\title{
The key to key presses: eSports game input streaming and copyright protection
}

\author{
Scott M. Kelly and Kirk A. Sigmon*
}

\begin{abstract}
The eSports industry has exploded, in no small part due to the ease with which exciting matches may be watched. Many modern video games stream game user inputs to viewers, rather than bandwidth-intensive video. These game input streams can be used by the viewers' game clients to perfectly reproduce a match in real-time. In World Chess U.S. v. Chessgames Services, a U.S. District Court held that allegedly pilfered chess game moves, as facts, were neither subject to copyright protection nor eligible for 'hot news' misappropriation. But might video game input streams (as facts, per the World Chess court) nonetheless be eligible for copyright protection to the extent that the input data corresponds to a copyright-eligible game performance? After all, input streams are significantly more granular and exacting than mere chess game moves: they capture millisecond-by-millisecond input and effectuate perfect reproducibility of gameplay, rather than a mere description thereof. This article explores the copyright issues under U.S. law presented by live streaming of video game inputs and proposes that video game input streams are, to the extent that they are usable to perfectly generate a faithful recreation of a gameplay performance, copyright-eligible and owned by the player of the game.
\end{abstract}

Keywords: eSports, user input, streaming, video games, game replays, copyright, hot news, World Chess

\section{Introduction}

It is well-established that facts are ineligible for copyright protection in the United States. ${ }^{1}$ In an attempt to avoid this principle, many plaintiffs have argued that the socalled 'hot news' doctrine provides a unique remedy for misappropriation of 'hot' facts wherein efforts were taken to collect those facts, and the value in those facts is in their newness or recent discovery. ${ }^{2}$ But the hot news' doctrine is limited at best, applicable only to the narrowest of circumstances. ${ }^{3}$ For example, in World Chess US, Inc. v. Chessgames Services LLC, No. 1:16-CV-08629 (S.D. N.Y. November 11, 2016) ('World Chess'), the court found that 'it is well-established that sports scores and events, like players' moves in [a chess championship], are facts not protectable by copyright' or by the hot news doctrine. ${ }^{4}$

But what would the result of World Chess be if the 'facts' were significantly more complex and granular, such that they were not so readily described as facts in the first place? Rather than a finite number of possible moves per turn, what if a chess game involved a seemingly endless series of possible inputs and outputs on a

Attorneys with Banner \& Witcoff, Ltd in Washington, D.C. The authors would like to thank Ross Dannenberg for his helpful comments during preparation of this article.

1 See Feist Pubs., Inc. v. Rural Tel. Serv. Co., Inc., 499 U.S. 340, 344-45 (1991).

2 See Int'l News Serv. v. Associated Press, 248 U.S. 215 (1918). millisecond-by-millisecond basis? Rather than an 8 by 8 board with specified movement rules for each piece, what if the board permitted exponentially larger directions and degrees of movement, and allowed each player to move each millisecond? Returning to the sports world, what if a computer were capable of perfectly observing and replicating a sports performance, such as the exact movements of a baseball player down to an atomic level? Would such information remain copyright-ineligible? After all, while the act of shooting a three-pointer is almost certainly far from creative, the unique combination of movements and strategy implemented by (some) athletes may very well comprise something minimally creative, rather than a purely mechanical arrangement of movements.

These questions are not merely hypothetical, but precisely the concerns presented in the context of eSports. In many video games, recordings of gameplay input - often called 'replays' or 'demos' - are able to perfectly replicate a player's gameplay when executed by game software. As early as id Software's DOOM, such game input data has 
been saved and used in order to reproduce a player's past gameplay. Today, game input data can be used for broader purposes. For example, many extremely popular Multiplayer Online Battle Arena ('MOBA') and First Person Shooter ('FPS') games, like Valve Software's Dota 2 and Ubisoft's Tom Clancy's Rainbow Six Siege, stream popular eSports competitions by transmitting input data to viewers' game clients, in effect offloading the task of rendering gameplay onto viewers' computers. Viewers are thereby given a better ability to control their viewing experience (e.g. the ability to move a camera around the game field).

It seems extremely unlikely that input streams would be eligible for protection via the 'hot news' doctrine, as used in World Chess: as explored in this paper, the 'hot news' doctrine is all but nonexistent in the modern era. But are such input streams eligible for copyright protection under existing copyright law? And if so, who would own such a copyright? On one hand, it may seem strange that a player's series of key presses for the purpose of playing a video game may be an original work of authorship by that player embodying a 'modicum of creativity,' particularly in a legal regime where chess game moves are 'facts' ineligible for copyright protection. On the other hand, an author must only contribute something more than a 'merely trivial variation' to be creative for the purposes of creating copyrightable work, ${ }^{5}$ and gameplay presents countless opportunities for a player's creative expression in the pursuit of victory. While chess moves are easily described (e.g. 'knight to f3'), input information is not so easily described and is used merely as a tool for recreating gameplay output, similar to how a player piano roll triggers a series of piano keys to generate a musical performance. And, even if an individual key press or mouse movement might not be creative, the key presses and mouse movements made in a video game may, in combination, entail at least a modicum of creativity. After all, this very article was constructed using a series of key presses and mouse movements, and (hopefully) few would argue that it lacks at least a modicum of creativity.

5 See Alfred Bell \& Co. v. Catalda Fine Arts, Inc., 191 F.2d 99, 102-103 (2d Cir. 1951)

6 While this article exclusively analyzes United States law, similar arguments may be made worldwide, particularly given the global popularity of eSports.

7 See ESPN eSports, available at http://www.espn.com/esports/ (last accessed February 23, 2018).

8 Craig Smith, '48 Amazing Twitch Stats and Facts' (February 2018), By the Numbers (February 17, 2018), available at https://expandedramblings. com/index.php/twitch-stats/.

9 Ben Gilbert, 'Amazon's Streaming Service Twitch is Pulling in as Many Viewers as CNN and MSNBC', Business Insider (February 13, 2018),
This article argues the case that current United States copyright law ${ }^{6}$ grants limited copyright protection to video game players' input streams, such as those used in popular eSports games like Riot Games' League of Legends and Valve Software's Counter-Strike: Global Offensive. This article begins in Section 2 by discussing input streams and replays, including their use in popular eSports titles. Section 3 provides a review of copyright law, including the history of copyright protection over performances. Section 4 discusses the World Chess case and analyzes issues relating to the legal protection of gameplay information. Section 5 argues for limited copyright protection for perfectly reproducible input streams, such as those in video games. Section 6 concludes the article.

\section{2. eSports and the growing value of gameplay input data}

The growing value of input data in video games, particularly as used to stream eSports matches, has raised an interesting question: could such input streams be copyrightable? And if so, who owns them?

ESports has been growing worldwide both in popularity and in value. eSports is, in essence, competitive video game playing: eSports athletes play multiplayer games (usually popular titles like Ubisoft's Tom Clancy's Rainbow Six Siege or Valve Software's Dota 2) against one another in competitions, often for a prize. Such competitions are increasingly broadcast, including on ESPN. ${ }^{7}$ As of 17 February, 2018, popular game streaming website Twitch.tv ('Twitch') had over 100 million monthly unique visitors, 15 million daily active users, and 2.2 million monthly broadcasters. ${ }^{8}$ These viewership figures suggest that Twitch averages more viewers than some major news networks. ${ }^{9}$ North America hosts only $28 \%$ of eSports events; there were over 400 eSports events held worldwide in 2017, each with a prize pool exceeding $\$ 5,000 .{ }^{10}$ To even start an eSports franchise, some game companies are charging as much as $\$ 20$ million. ${ }^{11}$ Such franchise fees are but a pittance in view of the profitability of gaming as a whole, as global revenue for video games already exceeds that of the music industry

available at http://www.businessinsider.com/twitch-is-bigger-than-cnnmsnbc-2018-2.

10 Newzoo, 2017 Global Esports Market Report (2017), p. 19, available at http://strivesponsorship.com/wp-content/uploads/2017/02/Newzoo_Free_ 2017_Global_Esports_Market_Report.pdf.

11 Kurt Badenhausen, 'Esports Leagues Set To Level Up with Permanent Franchises', Forbes (October 3, 2017), available at https://www.forbes com/sites/kurtbadenhausen/2017/10/03/esports-leagues-grow-up-withpermanent-franchises/\#3fc85c6421d6. 
by $\$ 20$ billion, and the industry is rapidly approaching the profitability of the movie industry. ${ }^{12}$ The gaming industry is particularly proud of that fact: Activision recently released a press release bragging that Call of Duty: WWII made more money in its first three days of release than the theatrical box office openings of Thor: Ragnarok and Wonder Woman combined. ${ }^{13}$

Many video games are streamed live to the Internet in a manner similar to the way in which a live football game is streamed on the Internet. While popular eSports matches may be streamed by a broadcaster (e.g. ESPN), many gamers, whether part of eSports or not, elect to stream their own games. Gamers which stream their live gameplay on websites like Twitch (so-called 'streamers') use popular streaming tools such as Open Broadcaster Software ('OBS') ${ }^{14}$ to capture, encode, and broadcast their video game to the world. Streams generated using such tools are merely video streams, not dissimilar to those used to disseminate webcam video, television shows, or the like. Such streams are of increasingly high quality thanks to advances in streaming technology and worldwide bandwidth: the vast majority of Twitch streams are at least $720 \mathrm{p},{ }^{15}$ with $1080 \mathrm{p} / 60 \mathrm{fps}$ streaming recently made available. ${ }^{16}$

But conventional video streaming has limitations: sending a $720 \mathrm{p}$ or $1080 \mathrm{p}$ video stream to viewers around the world can be extremely bandwidth-intensive and inefficient. Twitch recommends that streamers provide at least $1800-2500 \mathrm{kbps}$ of bandwidth for optimal performance, ${ }^{17}$ but this only tells part of the story: this data must ultimately be transmitted to hundreds, if not thousands, of viewers worldwide. This extensive data use is increasingly a large part of overall Internet traffic: by 2021, video (including Internet video and IP video on demand) will make up a whopping $82 \%$ of Internet traffic. ${ }^{18}$

A perhaps better option for streaming, explored by some game companies in recent years, is to allow fans to watch video game matches using their own copy of

12 Nick Wingfield, 'In E-Sports, Video Gamers Draw Real Crowds and Big Money', The New York Times (August 30, 2014), available at https:// www.nytimes.com/2014/08/31/technology/esports-explosion-bringsopportunity-riches-for-video-gamers.html.

13 See Activision, 'Call of Duty: WWII Scores Over Half a Billion Dollar Opening Weekend', BusinessWire (November 8, 2017), available at http://www.businesswire.com/news/home/20171108005673/en/Call-DutyWWII-Scores-Billion-Dollar-Opening.

14 See Open Broadcaster Software, https://obsproject.com.

15 Nicolas Muesch, 'Analysis of the Characteristics and Content of Twitch Live-Streaming', Worcester Polytechnic Institute (March 2015), p. 25, available at http://web.cs.wpi.edu/ claypool/iqp/twitch-tv/report.pdf.

16 Noreen McInnis, 'Our team (and this dog) present to you: 1080p streaming on Twitch', Twitch.tv (March 29, 2017), available at https://blog. twitch.tv/1080p-streaming-support-on-twitch-fea5e1945ae1.

17 Nicolas Muesch, 'Analysis of the Characteristics and Content of Twitch Live-Streaming', Worcester Polytechnic Institute (March 2015), p. 26, available at http://web.cs.wpi.edu/ claypool/iqp/twitch-tv/report.pdf. the same game. Rather than sending a video stream to thousands of fans at once, game companies instead allow players and tournament organizers to stream games by broadcasting game data from individual game clients (e.g. the game clients of the players), which may be used by fans' computers (e.g. via their own game clients) to recreate the game. ${ }^{19}$ The benefits of this process are numerous: more efficient use of bandwidth, better video quality, and, of course, additional marketing and promotional opportunities for the game creator.

An example of the distinction between video streaming and input streaming illustrates the convenience of the latter. As noted above, a video stream may require $1800-2500 \mathrm{kbps}$ of upload bandwidth for optimal performance. ${ }^{20}$ Once received by a viewer, the viewer's computer may process and display the received video stream in the same manner that, for example, they might process and display a movie on Netflix. In contrast, for input streams, orders of magnitude less data need be used. For example, one player of Valve Software's Dota 2 reported a total of $57.86 \mathrm{MB}$ downloaded and $13.30 \mathrm{MB}$ uploaded for a 42 minute game, ${ }^{21}$ suggesting a transmission rate of slightly less than $4 \mathrm{kbps}$. In contrast, at absolute minimum and at lowest settings, Netflix requires $500 \mathrm{kbps}$ of bandwidth. ${ }^{22}$

Whether live (as a 'stream') or recorded and stored for later (as a 'replay'), such input streams provide an exact or near-exact replica of gameplay, albeit rendered through a video game client instead of multimedia software. ${ }^{23}$ As explained by Patrick Dickinson in Gamasutra:

[A] more elegant approach is to make use of the inherent predictability of computer software: The same sequence of operations performed on the same set of data will always produce the same result. It is reasonable to conclude that a sequence of game play may be precisely reproduced by recording only the initial state of the game, along with the player's inputs. The initial state can then be restored, and the recorded inputs

18 See Rani Molla, 'An Explosion of Online Video could Triple Bandwidth Consumption Again in the Next Five Years', Recode.net (June 8, 2017), available at https://www.recode.net/2017/6/8/15757594/future-internettraffic-watch-live-video-facebook-google-netflix.

19 See, e.g. Arthur Gies, 'Where and How to Watch The International 2017, Dota 2's \$24 Million Tournament', Polygon (August 7, 2017), available at https://www.polygon.com/2017/8/7/16105692/how-to-watch-the-interna tional-2017-ti7-dota-2-24-million-tournament.

20 See Nicolas Muesch, 'Analysis of the Characteristics and Content of Twitch Live-Streaming', Worcester Polytechnic Institute (March 2015), p. 26, available at http://web.cs.wpi.edu/ claypool/iqp/twitch-tv/report. pdf.

21 See 'How Much Bandwidth does a Dota Match Take?', Gamefaqs.com (2013), available at https://gamefaqs.gamespot.com/boards/610657-dota$2 / 65266053$.

22 'Internet Connection Speed Recommendations', Netflix.com, available at https://help.netflix.com/en/node/306.

23 See ibid. 
reapplied, to produce the same sequence of play. This solution is instantly more appealing because the amount of data that needs to be stored is much smaller. ${ }^{24}$

One caveat in implementing such a system is that the game must necessarily be deterministic - that is, the exact same inputs must produce the exact same output. ${ }^{25}$ While special effects and rendering may be varied (e.g. a game event may be viewed from a different perspective or on different graphical settings), key game aspects such as game physics, artificial intelligence, gameplay, and animations must be deterministic. ${ }^{26}$ After all, there would be little point in a replay that did not actually duplicate game events. This process is easier said than done: as detailed in a blog post by Auston Montville, developer of games such as Sportsball and Catdate, ${ }^{27}$ implementing determinism in engines like Unity requires, among many other steps, re-programming various engine functions to be purely deterministic. ${ }^{28}$

Such input-deterministic functionality isn't exactly new. id Software's DOOM and DOOM II, released in 1993 and 1994 respectively, had the ability to record and reproduce game replays using recorded user input. ${ }^{29}$ Interestingly, in order to ensure determinism but still give the impression that certain aspects of the game (e.g. demon movement) were random, games like DOOM based events that appeared to be random on user input, such that the same exact sequence of input would result in the same 'random' event(s). ${ }^{30}$

Most major competitive eSports titles have some form of input recording and/or input streaming functionality. Riot Games' League of Legends uses proprietary (.rofl) files to store replays which may be viewed and converted into video files in-client. ${ }^{31}$ Valve Corporation's Source engine, used by both Dota 2 and Counter-Strike: Global Offensive, uses Source demo (.dem) files which 'basically store ... packets right off the wire. ${ }^{32}$ Similarly, both

24 Patrick Dickinson, 'Instant Replay: Building a Game Engine with Reproducible Behavior', Gamasutra (July 13, 2001), available at https://www.gama sutra.com/view/feature/131466/instant_replay_building_a_game_php.

25 Cyrille Wagner, 'Developing Your Own Replay System', Gamasutra (February 4, 2004), available at https://www.gamasutra.com/view/feature/ 2029/developing_your_own_replay_system.php.

26 Ibid.

27 Catdate is a 'game where you play as a cat and go on dates with other cats'. 'CatDate', AustonMontville.com, available at http://austonmontville.com/ catdate.html (last visited November 11, 2017).

28 See Auston Montville, 'Implementing a Replay System in Unity and How I'd Do it Differently Next Time', Gamasutra (November 5, 2014), available at https://www.gamasutra.com/blogs/AustonMontville/20141105/229437/ Implementing_a_replay_system_in_Unity_and_how_Id_do_it_differently_ next_time.php.

29 'About LMP/Demo Files', ClassicDoom.com, available at http://classic doom.com/lmpintro.htm (last visited November 11, 2017).

30 Ibid.

31 Mr Burnaby, 'Replays are Live in the New Client!: FAQ \& Pro-Tips', LeagueofLegends.com (November 16, 2016), available at https://boards. games have the ability to stream game data corresponding to game inputs, rather than broadcast video data. ${ }^{33}$ Such recording appears to be input-device agnostic such that, for example, there is no meaningful difference between recording an input stream of a game controller versus that of a mouse, a keyboard, or the like. Of course, such game data need not necessarily correspond to precise keystrokes or mouse movements - for instance, it appears that Valve's Source engine's .dem files store input data as transmitted in network messages; that is, the sort of data that might be received by a game server and from a game client. ${ }^{34}$

This trend raises an interesting question, as will be explored below: is the content in replay files, such as .rofl or .dem files, copyrightable? Is there copyright in a collection of keystrokes and mouse clicks generated by a player during a game?

\section{U.S. copyright law and the hot news doctrine}

To evaluate whether there may be copyright in video game activity, we must first discuss what copyright does - and does not - protect.

Copyright law protects 'original works of authorship fixed in any tangible medium of expression ... from which they can be perceived, reproduced, or otherwise communicated, either directly or with the aid of a machine or device.' 35 The 'original' requirement requires (1) independent creation and (2) a 'modicum of creativity.' ${ }^{36}$ The 'modicum of creativity' bar is quite low: an author must merely contribute a 'trivial' variation, ${ }^{37}$ and courts refuse to judge artistic merit. ${ }^{38}$ Original 'works of authorship' may include, among other things, pantomimes and choreographic works, motion pictures and other audiovisual works, and sound recordings. ${ }^{39}$

na.leagueoflegends.com/en/c/developer-corner/2JWmR6sF-replays-arelive-in-the-new-client-faq-pro-tips.

32 'DEM Format', Valve Developer Community, available at https://developer. valvesoftware.com/wiki/DEM_Format (last visited November 11, 2017).

33 See 'Spectating', The Dota 2 Wiki, available at https://dota2.gamepedia. com/Spectating; Spectator, The Counter-Strike Wiki, available at http:// counterstrike.wikia.com/wiki/Spectator.

34 See 'CS:GO Demos and Network Messages', Github (April 23, 2016), available at https:/github.com/ValveSoftware/csgo-demoinfo/blob/master/ demoinfogo/README.md.

3517 U.S.C. $\$ 102(a)$

36 Robert P. Merges, Peter Seth Menell and Mark A. Lemley, Intellectual Property in the New Technological Age (6th ed., Aspen Law \& Bus., 2012), p. 421.

37 Alfred Bell \& Co. v. Catalda Fine Arts, Inc., 191 F.2d 99, 102-103 (2d Cir 1951).

38 Bleistein v. Donaldson Lithographing Co., 188 U.S. 239, 251-52 (1903)

39 Ibid. 
The owner of such a copyright has the right, in the case of literary, musical, dramatic, and choreographic works, pantomimes, and motion pictures and other audiovisual works, to perform the copyrighted work publicly. ${ }^{40}$ The phrase 'fixed in a tangible medium of expression' need not imply perfect reproducibility: dance choreographers, for example, routinely use dance notation, film, and/or computer software to meet the fixation requirement. ${ }^{41}$

But copyright law explicitly does not protect 'any idea, procedure, process, system, method of operation, concept, principle, or discovery, regardless of the form in which it is described, explained, illustrated, or embodied. ${ }^{32}$ Perhaps most importantly for the purposes of this paper, facts are not copyrightable. ${ }^{43}$ After all, '[w]ere an author able to prevent subsequent authors from using concepts, ideas, or facts contained in his or her work, the creative process would wither. ${ }^{34}$

Admittedly, the distinction between a fact and copyright-eligible subject matter can be blurry. A creative compilation of facts, such as a uniquely-edited telephone directory, may be copyright-eligible, whereas the underlying facts (e.g. a particular person's telephone number) are not. ${ }^{45}$ The rationale is, among other considerations, that 'facts are not created or originated by the author of any work which embodies the fact. ${ }^{36}$ As such, courts have devised various tests to distinguish between creative compilations of facts and mere facts themselves, ${ }^{47}$ and factual compilations may be copyrightable. ${ }^{48}$ While a full discussion of how facts are defined under United States copyright law is beyond the scope of this paper, it suffices to say here that the line is blurry at best, with substantial emphasis placed on how a creator might use facts to create a work. ${ }^{49}$

A commonly-asserted exception to the concept that facts are not copyrightable is the so-called 'hot-news exception' to copyright law, which originated in International News Service v. Associated Press, 248 U.S. 215 (1918) ('International News'). In International News, the Associated Press ('AP') alleged that the International

40 Ibid at 106(4).

41 Krystina Lopez de Quintana, Comment, 'The Balancing Act: How Copyright and Customary Practices Protect Large Dance Companies Over Pioneering Choreographers' (2004) 11 Vill. Sports \& Ent. L.J. 139, 150.

4217 U.S.C. 102(b)

43 See Harper \& Row Publishers, Inc. v. Nation Enterprises, 471 U.S. 539, 547 (1985) ('no author may copyright facts or ideas').

44 Ibid at 582.

45 See Feist Pubs., Inc. v. Rural Tel. Svc. Co., Inc., 499 U.S. 340 (1991).

46 Howard B. Abrams, 'Originality and Creativity in Copyright Law' (Spring 1992) 55 L. \& Contemp. Probs. 7-8.

47 See ibid

48 See generally Feist Pubs., Inc. v. Rural Tel. Svc. Co., Inc., 499 U.S. 340 (1991).

49 See generally Howard B. Abrams, 'Originality and Creativity in Copyright Law’ (Spring 1992) 55 L. \& Contemp. Probs. 7-8.

News Service ('INS') was, among other things, unlawfully stealing the news from the AP. ${ }^{50}$ While the AP alleged that the INS committed a number of unlawful acts to pirate their news, the INS appealed but one issue to the Supreme Court: whether the INS could be lawfully restrained from copying news from public AP bulletin boards. ${ }^{51}$ Or, as stated by the Supreme Court, the issue was whether 'one who has gathered general information or news at pains and expense for the purpose of subsequent publication through the press has such an interest in its publication as may be protected from interference. ${ }^{52}$ The Court ruled that some protection did attach, thereby creating the hot news doctrine. ${ }^{53}$

The Court's ruling in International News Service was based not on theory of copyright, but as a 'quasi property' right rooted, at least in part, in unfair competition law. ${ }^{54}$ As noted by the Court, a 'news element - the information respecting current events contained in the literary production - is not the creation of the writer, but is a report of matters that ordinarily are publici juris; it is the history of the day. ${ }^{55}$ But the Court noted 'peculiar value' in 'spreading [the news] while it is fresh,' and that such value 'cannot be maintained by keeping it a secret. ${ }^{56}$ As such, the question was not one of copyright, but one of 'unfair competition in business;' that is, 'not so much the rights of either party as against the public but their rights as between themselves. ${ }^{57}$ The Court could accordingly 'hardly fail to recognize' that the news 'must be regarded as quasi property, irrespective of the rights of either as against the public. ${ }^{58}$ In other words, while the AP did not necessarily have a copyright to the 'history of the day,' it had a quasi-property right in the 'hot news' it acquired and disseminated such that the INS could not simply pilfer it from bulletin boards.

Since International News and after numerous changes in United States copyright law, little remains of the hot news doctrine. Indeed, some variant of the hot news misappropriation doctrine is only recognized in five states. ${ }^{59}$

50

51 Ibid.

52 Ibid

53 Ibid at 216

54 Ibid at $235-236$.

55 Ibid at 234

56 Ibid at 235 .

57 Ibid at 236

58 Ibid.

59 Kimberly Isbell, Citizen Media Law Project, The Rise of the News Aggregator: Legal Implications and Best Practices (2010), p. 16, available at http:// papers.ssrn.com/sol3/papers.cfm?abstract_id=1670339; see also John C. McDonnell, 'The Continuing Viability of the Hot News Misappropriation Doctrine in the Age of Internet News Aggregation' (2012) 10 Nw. J. Tech. \& Intell. Prop. 255. 
Even in those jurisdictions, the doctrine is extremely limited. For example, in National Basketball Association v. Motorola, Inc., 105 F.3d 841 (2d Cir. 1997), a case relating to Motorola's alleged misappropriation of NBA sports scores, the Second Circuit limited the hot news doctrine by providing an exceedingly narrow test for elements of a hot news claim:

In our view, the elements central to an [International News] claim are:

(i) the plaintiff generates or collects information at some cost or expense;

(ii) the value of the information is highly time-sensitive;

(iii) the defendant's use of the information constitutes freeriding on the plaintiff s costly efforts to generate or collect it;

(iv) the defendant's use of the information is in direct competition with a product or service offered by the plaintiff;

(v) the ability of other parties to free-ride on the efforts of the plaintiff would so reduce the incentive to produce the product or service that its existence or quality would be substantially threatened. ${ }^{60}$

A more recent Second Circuit case on the subject, Barclays Capital Inc. v. Theflyonthewall.com, Inc., 650 F.3d 876 (2nd Cir. 2011), reveals the difficulties in enforcing the 'hot news' doctrine in the modern age. In Barclays, defendant Theflyonthewall.com ('Fly') - an aggregator of the recommendations of security firms - used a variety of techniques to acquire securities recommendations issued by Barclays. ${ }^{61}$ The Second Circuit found that the majority of Barclays' claims were preempted by copyright law and were therefore not amenable to a claim of hot news misappropriation and that, in any event, Fly was not free-riding: 'the Firms seek only to protect their Recommendations, something they create using their expertise and experience rather than acquire through efforts akin to reporting ... Fly, having obtained news of a Recommendation, is hardly selling the Recommendation 'as its own. ${ }^{62}$ The Second Circuit provided a counterexample of activity that would give rise to a hot news misappropriation claim:

[i]f a Firm were to collect and disseminate to some portion of the public facts about securities recommendations in the

60 Nat'l Basketball Assoc. v. Motorola, Inc., 105 F.3d 841, 845 (2d Cir. 1997) (citations omitted).

61 Barclays Capital Inc. v. Theflyonthewall.com, Inc., 650 F.3d 876, 878-80 (2nd Cir. 2011).

62 Ibid at 905-906.

63 Ibid.

64 See, e.g. John C. McDonnell, 'The Continuing Viability of the Hot News Misappropriation Doctrine in the Age of Internet News Aggregation' (2012) 10 Nw. J. Tech. \& Intell. Prop. 255.

65 Victoria Smith Ekstrand and Christopher Roush, "From "Hot News" to "Hot Data": The Rise of "Fintech," the Ownership of Big Data, and the Future of the Hot News Doctrine' (2016) 35 Cardozo Arts \& Ent. 303. brokerage industry ... and were Fly to copy the facts contained in the Firm's hypothetical service, it might be liable. ${ }^{63}$

The 'hot news' doctrine has nonetheless been a favorite of commentators identifying flaws in modern copyright law, in effect using it as an escape valve for perceived inequity. For example, numerous articles have been written arguing for and against the applicability of the hot news doctrine to Internet news aggregation. ${ }^{64}$ Still others have argued that the doctrine could extend to so-called 'Big Data' in the finance industry to prevent smaller financial firms from 'scraping' financial data. ${ }^{65}$ Where copyright tends to fail, commentators look to the hot news doctrine to protect authors from practices perceived to be unfair.

\section{World Chess and the protectability of game moves}

The chess world has seemingly brought back discussion of the 'hot news' doctrine, albeit with an interesting twist: asserting the 'hot news' doctrine to protect game moves.

In World Chess US, Inc. v. Chessgames Services LLC, No. 1:16-CV-08629 (S.D.N.Y. November 11, 2016), plaintiff World Chess, a company in the business of organizing chess tournaments, sued various website competitors to prevent the alleged hot news misappropriation of a November 11, 2016 chess tournament. ${ }^{66}$ The defendants, who operated websites like Chess24. com, would copy and replay, in real time, chess moves made in tournaments such as those organized by World Chess. ${ }^{67}$ Chess 24 and the other defendants did not copy live video, commentary, or any copyrightable material besides the chess moves themselves. ${ }^{68}$ For viewers interested in merely seeing a chessboard and moves in real time, defendants thereby, arguably, provided a substantially similar experience to that of World Chess. ${ }^{69}$ A comparison of Chess24's chess viewer and World Chess's viewer is provided below. ${ }^{70}$

Per World Chess, such activity 'constitut[ed] free riding on World Chess's significant and costly efforts to organize, publicize, and disseminate major chess tournaments. ${ }^{31}$
66

World Chess US, Inc. v. Chessgames Services LLC, No. 1:16-CV-08629, Complaint at 2 (November 7, 2016).

67 Ibid at $8-10$

68 See generally World Chess US, Inc. v. Chessgames Services LLC, No. 1:16CV-08629, Mem. Op. at 3 (November 22, 2016).

69 See ibid.

70 See generally ibid at 10-11 (discussing alleged duplication by defendants). 71 Ibid at 11 

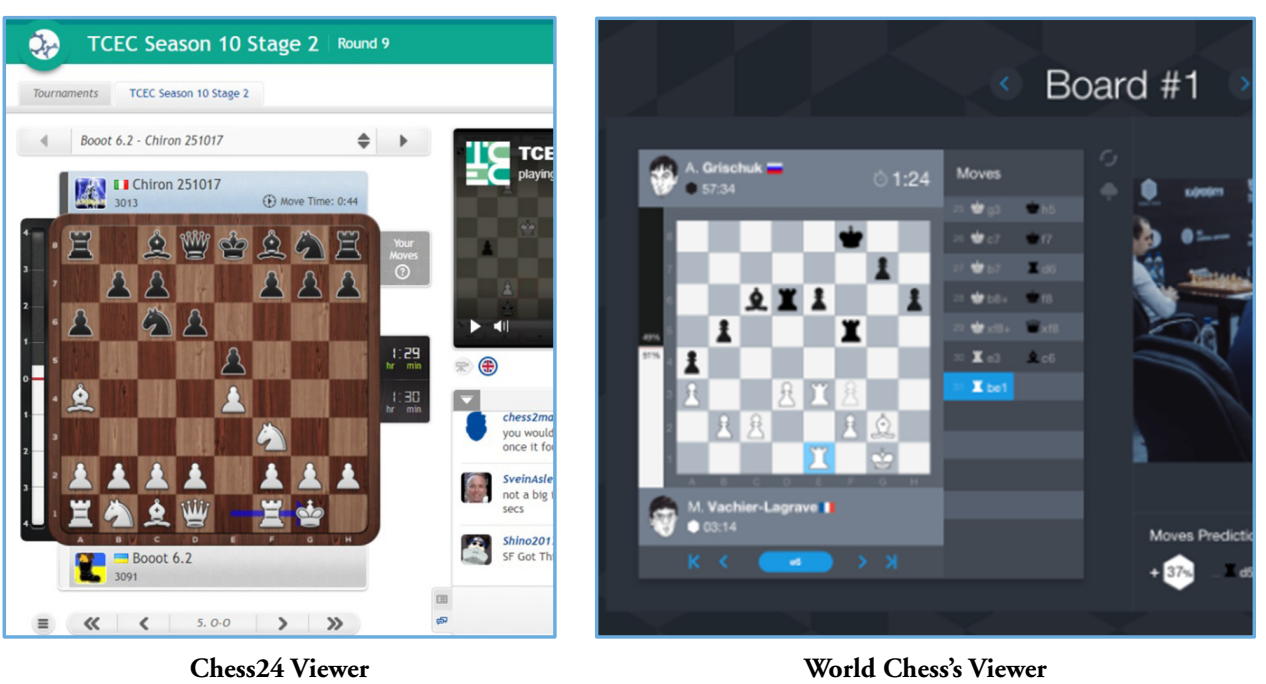

Figure 1 A comparison of Chess24's chess viewer and World Chess's viewer

In a decision and order issued November 22, 2016, District Judge Victor Marrero of the Southern District of New York rebuked World Chess's free-riding allegations: 'the evidence presented indicates that Chess 24 digests factual information about the Championship from secondary sources and creates its own website content at great expense. ${ }^{72}$ Citing National Basketball Association and Barclays Capital, the Court found that it is well-established that sports scores and events, like players' moves in the Championship, are facts not protectable by copyright. ${ }^{173}$

World Chess may have been a relatively easy case given the facts, but one suggesting a more difficult and interesting question: what if the 'facts' (that is, the chess moves) had been significantly more granular? Could there be some form of gameplay, whether physical or digital, that lends itself to recording movements so intricate and specific such that they capture the copyrightable aspects of a performance, rather than the mere facts thereof? Chess moves are simplistic; the process of describing chess moves (called chess notation ${ }^{74}$ ) is simple enough to perform verbally. In contrast, the intricate details of physical movement in a sport, such as the exact movement of every muscle in a baseball player's body, are far too complex to be accurately described using any form of notation. What if a recording of such physical movement could be made, to the extent that it was perfectly captured in every respect?

The concept of perfectly duplicating a creative physical performance may best be explained by imagining a hypothetical basketball game. As noted above, in National Basketball Association v. Motorola, Inc., the Second Circuit acknowledged a 'general understanding' that 'athletic events were, and are, uncopyrightable"75 and held that 'factual information culled from broadcasts' were not amenable to copyright protection. ${ }^{76}$ Indeed, it seems fairly uncontroversial that sports scores and facts, such as the fact that a player shot a 3-pointer at a certain time, are not amenable to copyright protection. Further, even extremely granular facts - such as 'Michael Jordan placed his left foot on the free throw line at 9:31:02 PM EST' - seem unlikely to be subject to copyright protection. But what if Michael Jordan's exact movements could be recorded and duplicated to an atomic level of specificity? Imagine, for example, a supercomputer and array of sensors configured to exactly record every single miniscule movement of Michael Jordan, such that every move could be reproduced, to an atomic degree, in a simulation. ${ }^{77}$ Would such granularity and specificity rise above mere 'fact' and become a copyright-protected fixture of his performance, such that exact reproduction of the performance

\footnotetext{
72 Ibid at 7.

73 Ibid at 9.

74 See 'Understanding Chess Notation', Dummies.com, available at http:// www.dummies.com/games/chess/understanding-chess-notation.

75 This is because "[s]ports events are not "authored" in any common sense of the word' such that, despite preparation, 'athletic events are competitive and have no underlying script'. Nat'l Basketball Assoc., 105 F.3d at 845-847.

76 Nat'l Basketball Assoc., 105 F.3d at 845, 847.
}

77 This level of perfect granularity is, admittedly, all but impossible. See Zohar Ringel and Dmitry Kovrizhin, 'Quantized Gravitational Responses, the Sign Problem, and Quantum Complexity' (2017) 3:9 Science Advances at 1. Amusingly, this article is often cited as 'proof that we do not live in a The Matrix-style simulation. See Anthony Cuthbertson, 'Are We Living in a Computer Simulation? Scientists Prove Elon Musk Wrong', Newsweek (October 4, 2017), available at http://www.newsweek.com/are-we-livingcomputer-simulation-scientists-prove-elon-musk-wrong-677251. 
therefrom were copyright infringement? After all, there is a significant difference between describing the broader facts of a performance (e.g. 'The actor walked to stage left') versus capturing minute details enabling an exact or near-exact replica of the performance (e.g. the atomic-level motion capture set forth above). Would a company infringe Michael Jordan's copyright in his performance $^{78}$ if it made available this fine-grained information, enabling an exactly perfect $3 \mathrm{D}$ rendered reproduction of his gameplay performance? ${ }^{79}$

This question may depend on how a copyright-ineligible 'fact' is defined, but such a bar appears easily overcome. Even if one were to construe all inputs as facts, courts must 'focus on the manner in which ... collected facts have been selected, coordinated, and arranged. ${ }^{80} \mathrm{In}$ other words, while a few repeated presses of a ' $W$ ' key on a keyboard seems far from creative, a fair argument may be made that a lengthy series of key presses effectuating a complex in-game performance would be remarkably different and might more likely entail a significantly greater degree of creativity, however minimal. ${ }^{81}$

Moreover, this question admittedly goes far beyond the 'hot news' doctrine. As discussed above, the 'hot news' doctrine is extremely fact-specific and limited, and the underlying motivation of preventing free-riding from collected facts seems inapplicable. And, while cases like World Chess may have purported to use the hot news' doctrine to protect game moves, such a strategy seems ineffective. Rather, the question presented herein is simpler: whether existing United States copyright law might arguably protect such input streams already.

The aforementioned question is not intended to suggest that the court in World Chess was incorrect. Rather, it is to suggest that there may be a spectrum such that particular forms of game capture and reproducibility may be uniquely copyright-eligible and that rights in such performances belong to the performers (that is, the players of the game). Game scores and championship results, for instance, may be at one side of the 'fact-versuscopyrightable' spectrum: few would argue they would ever be amenable to copyright protection. Somewhere in the middle of such a spectrum may be gameplay information from chess games and poker games, wherein description of discrete moves is easy and the number of moves themselves are significantly limited. World Chess and similar cases have held, and we do not dispute, that such information is not copyright-eligible. At the very end of such a spectrum might be eSports or the aforementioned supercomputer, wherein extremely complex gameplay facts ${ }^{82}$ may be captured, measured, and perfectly duplicated. Such input data may, like source code input to a compiler, perfectly create the same output in every instance. This extreme end of the spectrum is uniquely amenable to copyright protection, relative to other, lower-fidelity forms of gameplay and/or game reporting, such as the game moves in World Chess. In other words, the granularity offered by an input stream, as fixed in a tangible medium, is sufficient to fully capture the creative expression and is thereby copyright-eligible, just like the piano rolls of old. ${ }^{83}$

\section{(i). Copyrightability of gameplay performances}

Even assuming that technology exists to perfectly capture every movement of Michael Jordan, it is not immediately obvious that Michael Jordan would have a copyright in his activity during a basketball game. After all, a player (usually, and sometimes capriciously) 'performs' based on the rules of the game being played. Formula One driver Sebastian Vettel arguably does not seek to drive his car to be original, he drives his car to be faster than his competition. At least in theory, Sebastian Vettel's driving should not represent anything original or creative, but rather a human-limited attempt at driving perfectly given vehicle limitations, the properties of the track being raced on, the presence of other drivers, and the like. The converse applies as well: a touchdown dance performed by an American football player is arguably a creative work entirely unrelated to (and, sometimes, in violation of) game rules. ${ }^{84}$ And, in any event, even if such performances were amenable to copyright,
78 Assuming he owns it. See discussion infra regarding ownership issues.

79 We concede that this circumstance is all but impossible to ever occur, particularly in view of the existing U.S. copyright law regime, National Basketball League contracts and the work-for-hire doctrine, and the like.

80 Feist Pubs., Inc. v. Rural Tel. Svc. Co., Inc., 499 U.S. 340, 358 (1991).

81 Admittedly, one may dispute what a 'fact' in a video game is. For instance, the fact may be the input itself, the output as effectuated by the game, or some combination of both. This article does not explore this topic in depth, but rather focuses exclusively on key presses.

82 This question may be, of course, very different depending on the game in question, such that games with extremely limited input schemes may prevent creative expression. For example, Activision's Dragster for the Atari 2600 only allows the player to shift gears, only from the first to fourth gear (in that order), and only under specific conditions. See 'Activision Dragster Instructions', Atari Age, available at https://atariage.com/manual_html page.php?SoftwareLabelID $=1050$. For such a title, it seems unlikely that any set of input could be creative. Indeed, the range of input in Dragster is so finite that it was used to discover that a 35 -year-old world record for the title was false. See Amy B. Wang, 'A Man Accused of Cheating at Video Games May Lose His Guinness World Record', The Wash. Post (January 29, 2018), available at https://www.washingtonpost.com/news/ early-lead/wp/2018/01/29/a-man-accused-of-cheating-at-video-gamesmay-lose-his-decades-old-guinness-world-record/?utm_term=.1795a97e51cb.

83 See White-Smith Music Pub. Co. v. Apollo Co., 209 U.S. 1 (1908).

84 Note also that it is explicitly identified by the U.S. Copyright Office as 'not protected by Copyright', but legal argument supporting such a conclusion 
a player's copyright in their performance is likely best considered a work-for-hire for their respective team.

Regrettably, the U.S. Copyright Office is far from clear in defining what type of physical activity, as choreography, may be copyright-eligible. Take, for example, a quintessentially creative activity: dance choreography. Choreography, the 'composition and arrangement of a related series of dance movements, ${ }^{, 85}$ is explicitly amenable to copyright protection. ${ }^{86}$ But the amenability of choreography to copyright is limited: the Copyright Office has stated that '[r]egistrable choreographic works are typically intended to be executed by skilled performers before an audience' such that 'celebratory end zone dance move[s],' 'simple routines,' 'social dances' are not 'distinct works of authorship,' even if they may 'contain a substantial amount of creative expression. ${ }^{87}$ In other words, the U.S. Copyright Office views the originality of a work in terms of the sum of its parts: '[a] compilation of social dances, simple routines, or other uncopyrightable movements may not qualify as a compilation if the author's selection, coordination, or arrangement of steps or movements does not result in an expressive or compositional whole that constitutes copyrightable subject matter. ${ }^{.88}$ Unfortunately, this statement does little but beg the question of what defines an 'expressive or compositional whole that constitutes copyrightable subject matter. ${ }^{\prime 89}$ After all, what if the celebratory end zone dance move were extensively choreographed beforehand? ${ }^{90}$ What defines the line between a 'simple' and 'complex' dance routine? When is a dance, even a social dance using a fixed number of dance moves, not at least minimally creative?

Independent of the lack of clarity at the U.S. Copyright Office, game play performances associated with video games seem precisely the kind of material which should be protected by copyright for several reasons.

First, performances - including video game performances - are almost certainly 'original works of authorship.'

is not provided. See 'Copyright Registration of Choreography and Pantomime', U.S. Copyright Office (September 2017), p. 3, available at https:// www.copyright.gov/circs/circ52.pdf. Others have argued that such moves should be eligible for copyright protection. See, e.g. Hennry M. Abromson, 'The Copyrightability of Sports Celebration Moves: Dance Fever or Just Plain Sick?'’ (2004) 14 Marq. Sports L. Rev. 571.

85 'Copyright Registration of Choreography and Pantomime', U.S. Copyright Office (September 2017), p. 1, available at https://www.copyright.gov/ circs/circ52.pdf.

86 Ibid at $1-2$.

87 Ibid at 3 .

88 Ibid at 4.

89 See ibid. It also seems to fundamentally misunderstand ballroom dancing. Indeed, U.S.-based ballroom dance competitions outside the so-called syllabus level allow any movement or choreography desired except for lifts. See USA Dance, Inc., Dancesport Rulebook (2017), pp. 49-50, available at http://documents.usadance.org/governance/rules-policies-and-bylaws/ w4nctuekwe.
Though limited in a variety of dimensions (e.g. the control scheme of a video game), video game performances entail intentional movements which result in the authorship of some result, be it a 'Play of the Game' in Blizzard Entertainment's Overwatch or movement entirely unrelated to a goal in a game in Riot Games' League of Legends. And, while such video game performance works must be 'independently created by the author, ${ }^{91}$ this does not suggest that video game performances are outright copyright-ineligible; rather, merely that such performances cannot be duplicates of other performances. ${ }^{92}$

Second, video game performances almost certainly have 'some minimal degree of creativity. ${ }^{93}$ The creativity requirement in copyright law is 'extremely low' such that even a 'slight amount' of creative expression will suffice. ${ }^{94}$ If a creative work can be generated on a piano keyboard, it stands to reason that a creative performance could be created using a keyboard, mouse, or gamepad, even if arguably limited by the rules of a game. ${ }^{95}$ And, while it may seem somewhat silly to suggest that all movements in League of Legends are creative, it seems almost equally silly to conclude that all series of movements in League of Legends lack even a 'minimal degree of creativity.' For instance, a player moving their character around a game field in an arbitrary manner to waste time may be creative, even if uninteresting. Indeed, the use of game input has been used to create entire movies (sometimes called 'Machinima'96), such as Rooster Teeth's 'Red Versus Blue. ${ }^{.97}$ In contrast, chess moves are arguably not particularly creative or expressive, but oftentimes reflect a selection of one of a plurality of options based on a player's strategic goals.

Third, video game performances are easily 'fixed in any tangible medium of expression.' Indeed, because video games are inherently digital, such fixation is in fact easier than the fixation of, say, a choreographed dance. As discussed above, gameplay input is readily recorded in video games and stored in computer files.

90 Indeed, others have argued that end zone dances should be copyrighteligible. See supra fn. 66.

91 Feist Pubs., Inc. v. Rural Tel. Svc. Co., Inc., 499 U.S. 340, 345 (1991).

92 That is, independent creation must exist. See ibid.

93 Brown v. Entm't Merchants Ass'n, 131 S. Ct. 2729 (2011), affg sub nom. Video Software Dealers Ass'n v. Schwarzenegger, 556 F.3d 950 (9th Cir. 2009).

94 Feist, 499 U.S. at 346.

95 If game rules limited input to an extreme degree, one might argue that some form of the merger doctrine applies. See generally Michael D. Murray, 'Copyright, Originality, and the End of the Scenes a Faire and Merger Doctrines for Visual Works' (2006) 58 Baylor L. Rev. 779.

96 See Martin Picard, 'Machinima: Video Game as an Art Form?', Canadian Game Studies Association, available at http://journals.sfu.ca/loading/ index.php/loading/article/viewArticle/17.

97 See 'Red Vs. Blue', Rooster Teeth, available at http://roosterteeth.com/ show/red-vs-blue. 
Even if such input data were translated into hex codes and recorded on paper, this analysis would not change: after all, 'it makes no difference what the form, manner, or medium of fixation may be.' ${ }^{98}$ Indeed, underpinning our conclusion herein that input streams should be copyright eligible lies a recognition that such recorded input streams may be one form of the requisite tangible fixation of the copyright-eligible gameplay.

\section{(ii). Ownership of copyright in game play performance}

We argue herein that ownership of such a limited copyright over input streams should vest in the player of the game itself. However, existing precedent does not necessarily lead to this result. In Micro Star v. FormGen, the Ninth Circuit held that third-party created custom maps for Duke Nukem 3D were infringing derivative works, and thus any copyrights would be held by the game's rights owner. This holding was based on findings that the maps had a definite form (that is, map files on a CD-ROM and/or the audiovisual displays generated therefrom) and that the maps supplanted Duke Nukem $3 D$ content. ${ }^{99}$ Input streams appear to, at least initially, be somewhat analogous to those Duke Nukem 3D maps: after all, as discussed above, such streams may be stored (and/or create audiovisual displays) and, at least theoretically, may be used to supplant eSports games. As such, one may argue that input streams are derivative works of video games as, indeed, players are arguably authorized to create non-infringing 'derivative works' vis-à-vis their input in a game creating gameplay output. ${ }^{100}$ But our argument need not entail derivative works. As discussed herein, the copyright protection of input streams should be limited to the input alone, rather than extending to the broader displays/output of the game itself. In other words, a video game player should have a limited copyright in their key presses as stored, for example, in packets, but only that - not, for example, a video recording of their gameplay that uses

98 H.R. Rep. No. 94-1476, at 52 (1976), reprinted in 1976 U.S.C.C.A.N. at 5666.

99 Micro Star v. FormGen, Inc., 154 F.3d 1107, 1111 (9th Cir. 1998); see also Ross Dannenberg, 'Case: Micro Star v. Formgen (9th Cir. 1998) [C]', Patent Arcade (July 16, 2007), available at http://www.patentarcade.com/ 2007/07/case-micro-star-v-formgen-9th-cir-1998.html.

100 The question of whether the end result of a video game (e.g. the ephemeral 'creative work' that is the execution of a video game in a particular gameplay session) is copyrightable may best be suited for a separate article.

101 See U.S. Const., Art I, Section 8, Cl. 8.

102 There is 'no dispute that video games are generally copyrightable', though some may dispute whether certain aspects of video games are copyrightable. See, e.g. Michael E. Davis v. Elec. Arts, Inc., No. 10-CV-03328-RS at *3-4 (N.D. Cal. December 11, 2017) (game play itself not fixed in a tangible medium of expression). For instance, some have argued that games, in-game assets, in the bare facts of a game (e.g. the score), or the like. That input stream (as network packets or otherwise) may only be useful in the context of the video game, but may enjoy independent copyright protection divorced from the video game played. The existence of a superior copyright in the rendered visuals vested in a game company should change little: even if the rights of a player in their input stream as separated from the game itself are extremely minimal, such ownership may be sufficient to provide necessary incentives to foster the continued growth of a diverse eSports scene.

Vesting ownership of input streams in game creators (and not players), as may be suggested by Micro Star, would accomplish little in terms of 'promot[ing] the progress of ... useful arts.' ${ }^{\prime 01}$ The creator of a game has an unquestioned right to many parts of a game (e.g. game art and music), such that input streams are likely only valuable to the extent permitted by the creator of the game. ${ }^{102}$ Indeed, any arguably derivative copyright based on a videogame is subject to the copyright of the game owner itself. For example, in Blizzard Entertainment v. Lilith Games (Shanghai) Co., the court reasoned that independently-submitted heroes to third party title DotA Allstars were no more than parts of the larger work that was Blizzard's Warcraft III (and the original Defense of the Ancients mod). ${ }^{103}$

In contrast, vesting ownership of input streams in players creating those input streams provides valuable protection for those players, particularly in the context of competing third party leagues. Viewers of eSports tournaments are not merely interested in the game itself (which, of course, they may play themselves), but in the gameplay of other players. While players may have little reason to complain that a game creator uses their input streams in various ways, such players may object to other players or websites using their input streams. Vesting ownership of a copyright in input streams in players may thereby provide those players valuable control over their gameplay: they may, at minimum, be able to control which third party eSports leagues use their input

standing alone, are systems and are therefore uncopyrightable. See Bruce E. Boyden, 'Games and Other Uncopyrightable Systems' (2011) 18 George Mason L. Rev. 439. This conclusion might suggest that input streams corresponding to such games are not subject to and/or derivative of the copyright of the game creator; however, such a conclusion might be of little value in practice: what good is an input stream without copyrightable assets, such as in-game art and music?.

103 Order Denying Motion for Partial Summary Judgment and Denying Motion for Rule 11 Sanctions, Blizzard Entertainment, Inc. v. Lilith Games (Shanghai) Co. Ltd., No. 3:15-CV-04084-CRB (N.D. Cal. May 16, 2017); see also Jennifer Lloyd Kelly, Nicholas A. Plassaras and Chieh Tung, 'Crowdsourced Content in Video Games: How Ownership Issues Almost "Ganked” a Copyright Case', Lexology (January 4, 2018), available at https://www.lexology.com/library/detail.aspx?g=38de7c41-c297-480398dc-7b7bc1091bdd. 
streams. Granting players this right may provide benefits to third party leagues as well, since they will be able to obtain an effective assignment of those rights and therefore be able to exclude competitors from copying streams of their competitions. This is quite dissimilar to traditional performances, which are not so readily duplicated: whereas an NFL ${ }^{104}$ player may rest safely knowing that, for example, Vince McMahon may never duplicate their in-game performance in the $\mathrm{XFL},{ }^{105}$ the same does not apply to an eSports player, where a competing league or website may very readily exactly duplicate their performances.

An analogy may be drawn to the piano roll cases of the early 20th century. In White-Smith Music Publishing Co. v. Apollo Co., the Supreme Court wrestled with the question of whether piano rolls - that is, rolls of perforated paper which made player pianos play musicinfringed the copyright of composers' sheet music. ${ }^{106}$ Like modern input streams, the piano rolls were merely sets of instruction which, when executed by a player piano, reproduced some creative work. While the Court found that the rolls were not 'copies within the meaning of the copyright act, ${ }^{107}$ such a finding is almost certainly mooted by the modern Copyright Act (which is significantly broader and format-agnostic). ${ }^{108}$ But, importantly, the Court's discussion focused on whether the piano roll would infringe copyright in the corresponding musical composition. The Court did not propose that the piano roll would infringe some intellectual property rights of the player piano-just the composition. In other words, the underlying content mattered more than the mechanism with which the content was reproduced.

In the context of our eSports discussion, input stream data is like a player piano roll, with game software more akin to a player piano than any protected expression infringed by the piano roll. Game software, like a player piano, may be subject to a wide range of intellectual property protection. But an input stream does not necessarily infringe the copyright of software: rather, it reflects the creative expression of a player using the software, much in the same way a piano roll reflects the creative expression of a composer rather than the manufacturer

104 Revealing our US origins, this is the National (American) Football League. 105 Which is, fortunately or unfortunately, soon to return. Kevin Draper, 'Vince McMahon Says He Will Revive the XFL, With a Very Different Look', The New York Times (January 25, 2018), available at https:// www.nytimes.com/2018/01/25/sports/football/vince-mcmahon-xfl.html. 106 White-Smith Music Pub. Co. v. Apollo Co., 209 U.S. 1, 8-9 (1908). 107 Ibid at 18 .

10817 U.S.C. $\$ 102$ (a) (which, unlike the applicable law at the time of WhiteSmith, provides that '[c] opyright protection subsists, in accordance with this title, in original works of authorship fixed in any tangible medium of expression') (emphasis added). of the player piano. In other words, input stream data comprises the creative and expressive performance of a player via a game, rather than the creative work of the game itself. Accordingly, like how a piano roll may entail the rights of the creator of a musical composition but not the rights of the creator of a player piano, input stream data may entail the rights of a player of a game but not the rights of the creator of a game.

\section{(iii). Enforcement of gameplay copyrights}

Assuming that game performances were amenable to at least a minimal form of copyright protection, would players or game companies thereby have a right to protect their input streams from unauthorized use, at least from other players or websites besides the game creator?

This question isn't theoretical: the gaming community has already begun developing tools which enable the analysis and replication of others' video game input recordings. A tool known as 'Baron Replays' once allowed League of Legends players to record and duplicate their games; however, as of this article, it appears that Riot Games has shut down the Baron Replays website and permanently banned many of the tool's users. ${ }^{109}$ Similarly, a user named Devilesk has developed a web browser-based tool to analyze Dota 2 replays. ${ }^{110}$ A user named AkiVer recently created a Counter-Strike: Global Offensive 'Demos Manager,' a tool which allows users to, inter alia, retrieve score data, player information, track grenades thrown, and generate heat maps of gameplay movements from game recordings. ${ }^{111}$ It seems almost a matter of time before more tools to analyze and utilize replays are developed-potentially a growing concern for game companies seeking to maintain close control over their games.

One might argue that the aforementioned tools violate other copyrights or trademarks, such as the rights in the game's artwork and other assets, and that an alleged copyright over an input stream need not be required for companies to protect their games and for players to protect their gameplay. But this perspective is myopic. It is no secret that games like Riot Games'

109 See Baron Replays, Facebook.com, https://www.facebook.com/baronrp. It appears that this may have been because Baron Replays enabled so-called 'zoom hacks'. See 'Baron Replays: Can it Get you Banned?', League of Legends Boards (2016), available at https://boards.na.leagueoflegends. com/en/c/GD/jNziEaTE-baron-replays-can-it-get-you-banned.

110 See Replay Viewer, Devilesk.com, http://devilesk.com/dota2/apps/replay/ viewer.

111 See Counter-Strike Global Offensive Demos Manager, available at https:// csgo-demos-manager.com. 
League of Legends, Valve Software's Dota 2, and other MOBA titles are in fierce competition. ${ }^{112}$ Moreover, as BlueHole Studio has recently learned vis-à-vis Epic Games' apparent duplication of the gameplay in Player Unknown: Battlegrounds, ${ }^{113}$ game genres and modes are far from protectable. What if a new entrant in the MOBA market independently developed a new game with entirely original artwork, maps, and characters which, when fed input data from Dota 2, produced almost exactly the same gameplay albeit using the new artwork, maps, and characters? Absent a copyright in the input stream data, these cloned games could recreate a player's expressive performance without fear of reprisal.

Relatedly, it is not necessarily the case that game software (or, in turn, a game creator's copyrights) need be involved to stream input. Numerous tools exist to record key strokes and mouse movement to, for example, generate heat maps of mouse movement. ${ }^{114}$ Should a player truly desire, they could track their gameplay inputs without ever involving gameplay software and using similar tools. As such, it is not necessarily the case that input data streaming requires permission from the game creator.

An example of a different type of tool that might not violate copyrights in the game itself but which may use gameplay input data is patented as U.S. Patent No. 7,500,916, 'Game Strategy Analysis Tool Generating a Two Dimensional Image Overlaid with Telemetry Data.' Per the patent:

Game session telemetry data corresponding to a first playercharacter is received from a game playing device. The first player-character is controlled by a user of the game playing device. The game session telemetry data is stored in a database for subsequent querying by a user. Based on the user query, a two dimensional image is generated, where the two dimensional image depicts a first view of the simulated environment overlaid with the queried telemetry data. The overlaid telemetry data is placed in the two dimensional image at an approximate location of each event to which the overlaid telemetry data corresponds. ${ }^{115}$

In other words, game events (e.g. from Bungie's Halo: Combat Evolved) are stored via game session telemetry data, and this data is later provided in a digestible

112 See 'Comparing MOBAs: League of Legends vs. Dota 2 vs. Smite vs. Heroes of the Storm', VentureBeat (July 15, 2015), available at https://venturebeat.com/ 2015/07/15/comparing-mobas-league-of-legends-vs-dota-2-vs-smite-vsheroes-of-the-storm.

113 See Kirk Sigmon, 'Epic sues alleged Fortnite hackers Brandon Broom and Charles Vraspir', Patent Arcade (October 11, 2017), available at http:// www.patentarcade.com/2017/10/epic-sues-alleged-fortnite-hackers.html.

114 See, e.g. Luke Plunkett, 'Tracking where a Mouse Moves while Gaming', Kotaku (April 25, 2015), available at https://kotaku.com/tracking-wherea-mouse-moves-while-gaming- 1706832273.

115 U.S. Pat. No. 7,500,916 col. 2 lines 48-58. format. Might a player (or, more likely, an eSports tournament organizer to whom the player has assigned rights) be able to object to his game play performance being utilized in such a manner by third parties? Could Bungie do anything about such analysis if it carefully avoided using Bungie's existing copyrights or trademarks? Absent some form of protection of input streams, the answer seems to be no.

\section{An argument for a limited extension of copyright}

A particularly strong argument may be made that, under existing United States copyright law, input streams are copyrightable, at least to the extent that such input streams correspond to a copyright-eligible video game performance. A legal regime to the contrary would accomplish little other than ignoring modern technological developments and potentially impeding the growth of the eSports industry.

As discussed above, input streams are increasingly being used to supplant and/or replace video streams and video recordings of eSports gameplay. ${ }^{116}$ Such video streams and video recordings of gameplay are almost unquestionably copyright-eligible - why then, should input information be any different? Should copyright truly hinge on whether one can watch gameplay via a H.264 video ${ }^{117}$ played in multimedia software or via an input stream in a game client?

A rational application of existing copyright law counsels for the protection of input streams, at least to the extent that they may perfectly reproduce extremely complex input which may not be so readily summarized as mere fact. By way of example, video game website Giant Bomb ${ }^{118}$ can remain free to report on facts that occurred in a video game (e.g. that Jeff Gerstmann got a 'Chicken Dinner' in Player Unknown's Battlegrounds ${ }^{119}$ ), but should not be free to compete with Valve Corporation's The International by selling the exact input data from the tournament as distributed by Valve.

A limited copyright over input streams need not suggest a broadened hot news doctrine (or, indeed,

116 See, e.g. Arthur Gies, 'Where and How to Watch The International 2017, Dota 2's \$24 Million Tournament', Polygon (August 7, 2017), available at https://www.polygon.com/2017/8/7/16105692/how-to-watch-the-interna tional-2017-ti7-dota-2-24-million-tournament.

117 For an overview of H.264 technology, see Sid Bala, 'H.264 is Magic', Sidbala.com (November 2, 2016), available at https://sidbala.com/h-264is-magic.

118 See generally 'Giant Bomb', http://www.giantbomb.com

119 See Luke Plunkett, 'The Origins of Battlegrounds' "Winner Winner Chicken Dinner" Line', Kotaku (July 13, 2017), https://kotaku.com/theorigins-of-battlegrounds-winner-winner-chicken-din-1796870568. 
application of the hot news doctrine at all), nor suggest that the reporting of gameplay information (e.g. a radio broadcast describing a football game) is copyright infringement of player performances. Rather, the key is that input data such as that used in Valve Software's Counter-Strike: Global Offensive allows for the exact duplication of gameplay, ${ }^{120}$ which itself may be a copyright-eligible performance. The fact that one player in Counter-Strike: Global Offensive shot another is far from copyright-eligible; an exact recording of every player's movement throughout a game is a different question.

That video gameplay input is, usually, a series of mouse movements and key presses cannot and should not defeat copyright eligibility. It might seem silly, for example, to argue that the press of a piano key is copyrightable, but a song - played on a piano and based on a series of key presses - is copyrightable, ${ }^{121}$ as is a recording of key inputs (e.g. a MIDI file) which, when processed by a computer, duplicates such key presses. ${ }^{122}$ Thus, to the extent that a game performance is at least minimally copyright-eligible, the fact that a game performance comprises and is recorded via a series of computer inputs cannot defeat copyright eligibility. As a type of recording in a tangible medium, sufficient to enable reproduction of the copyright-eligible performance, it is reasonable to extend copyright protection to the recorded input stream.

Of course, copyright protection over input data must be carefully limited to avoid abuse. Extending copyright protection to arbitrary input commands would scarcely benefit anyone and merely fill the world with possible infringement traps. For example, a professional player should not be able to claim a copyright over a particularly efficacious series of inputs that provide the player with an advantage, isolated from the rest of the gameplay performance. ${ }^{123}$ Admittedly, this may be a difficult line to draw, particularly with games with extremely limited input options (e.g. rudimentary platforming video games), and may suggest that the copyright in input streams should not be infringed by other players recreating even substantial parts of such a stream through their own inputs. The copyright eligibility of an input stream must, therefore, be no greater than the copyright eligibility of the performance captured, and that performance should be carefully scrutinized to prevent abuse.

And, whatever copyright there is in input streams is almost unquestionably subservient to the copyright interest in game assets themselves, in addition to applicable licensing agreements governing video games.
Needless to say, there would be little value in an input stream corresponding to Ubisoft's Tom Clancy's Rainbow Six Siege without some right to use or display Tom Clancy's Rainbow Six Siege itself, including the game's unique maps, characters, and the like. And, notwithstanding such copyright interests, Ubisoft could readily enforce licensing agreements and/or terms of service which required that player transfer their limited input stream copyright interests to Ubisoft.

Moreover, provision of some form of copyright to players does not impede the ability of game creators to develop, promote, and/or control third party leagues. A game creator's copyrights and terms of service already provide the game creator with the ability to control how a game may be used by third party leagues. As such, while a copyright in input streams may allow a player to prevent unauthorized third parties from using their input, their rights only go that far.

As already suggested above, a reasonable argument could be made that all gameplay, whether recorded or not, should simply be ineligible for copyright protection. The 'well-established' ${ }^{124}$ rule that sports scores and players' moves are not copyright-eligible arises at least in part from a recognition that significant harm would likely result from allowing monopolies over facts such the score of a local basketball game or a particular play in a football game. While this paper discusses the possibility that unauthorized individuals may use input stream data to provide competing video streams of popular eSports titles, it is admittedly more likely that input streams would be downloaded and used by passionate fans seeking to watch exciting eSports matches and learn new strategies. Should such a passionate fan be sued for copyright infringement if they download and watch an input stream of their favorite Dota 2 player without permission from that player or from the game developer? It seems like a bad idea, even from a public relations perspective, for a game company to unnecessarily limit their fans' ability to enjoy their games.

Furthermore, the likelihood that anyone could or would be capable of using input data to compete with an eSports game is all but de minimis. Assume, for example, that a nefarious company sought to compete with Valve Software's Counter-Strike: Global Offensive by duplicating the streams from popular eSports tournaments. What would said company do with the streams?
120 DEM Format, Valve Developer Community, available at https://developer. valvesoftware.com/wiki/DEM_Format (last visited November 11, 2017).

121 As, at least, a musical work. See 17 U.S.C. $\$ 102(2)$.

122 See, e.g. Glover v. Austin, 289 Fed. Appx. 430 (2nd Cir. 2008) (evaluating analogue reel tape and MIDI file similarly).
123 Indeed, to the extent such movements provide utility within the game, they might be copyright ineligible as being primarily functional in nature. 124 World Chess US, Mem. Op. at 9. 
Use of the streams along with in-game assets (e.g. in-game artwork or models) would infringe the copyright of the game assets themselves. ${ }^{125}$ Would players actually want to watch a game based on input streams from a popular video game but without the popular video game's assets by using, for example, some form of a third party program? Such a result seems unlikely. It seems significantly more likely that the gaming community would meet such an attempt at free riding with vitriol.

Encouraging, via copyright protection, companies to more freely broadcast input streams may have an added, more pragmatic benefit: network efficiency. Third party eSports leagues ${ }^{126}$ should not have to elect lossy and/or laggy video streaming to avoid the possibility that their competitors may acquire and misuse their input streams, particularly since, as discussed above, input streaming may be significantly more bandwidthefficient.

A limited copyright over input streams, as owned by players, may particularly help protect third party eSports leagues. Should, for example, a popular third party league develop around custom Counter-Strike: Global Offensive matches, it is possible that others (e.g. competing leagues) may seek to re-broadcast such matches through use of input streams. Protecting such input streams through copyright law and vesting such copyright in players may allow such leagues to better establish ownership over aspects of their matches-subject, of course, to the rights of the game creator. Such ownership may be necessary to make such competitions economically viable. Indeed, as game creators already have a wealth of rights (e.g. copyrights and trademarks) in their titles which they may use against infringers, third party leagues may be the primary beneficiaries of recognizing copyright protection in input streams. Absent such protection, a third party league with no exclusive relationship with the game creator may find themselves without any recourse when a rival site begins redistributing replays of their hosted games, à la World Chess.

During the authorship of this very article, a similar issue arose with regard to Valve Software's Dota 2. In late 2017 and early 2018, the Electronic Sports League (the 'ESL') made Facebook its main broadcast partner. ${ }^{127}$ As such, viewers would have to use Facebook's streaming services to watch certain ESL games, as the ESL-Facebook agreement allegedly prevented broadcasting on Facebook and Twitch simultaneously. ${ }^{128}$ The Dota 2 community, openly skeptical of this partnership, began to use Twitch to broadcast ESL's games (as reproduced in their game client, rather than via Facebook) instead. ${ }^{129}$ The ESL responded by sending out Digital Millennium Copyright Act ('DMCA') takedown notices to the Twitch streamers, asserting ownership of the games and the corresponding streams. ${ }^{130}$ Valve was forced to step in, asserting its ownership of Dota 2:

The first issue we've been seeing discussed is regarding DMCA notices. This one is very simple: No one besides Valve is allowed to send DMCA notices for games streamed off of DotaTV that aren't using the broadcasters' unique content (camera movements, voice, etc).

The second issue is regarding who is permitted to cast off of DotaTV. We designed the DotaTV guidelines to be flexible in order to allow for up and coming casters, or community figures like BSJ or Bulldog that occasionally watch tournament games on their channel, to be able to stream off of DotaTV. It is not to allow commercial organizations like BTS to compete with the primary stream. It'll be our judgment alone on who violates this guideline and not any other third party's. ${ }^{131}$

While Valve Software's response appears to have calmed the concerns of Dota 2 streamers for the time being, the issue remains: game leagues need protection from their competitors, even if their copyrights are indeed subject to the copyrights of, for example, game developers.

In sum, if input streams were not amenable to copyright protection, the eSports industry - and, in particular, third party eSports leagues - may be inhibited or outright harmed. Without a clear delineation of ownership as to input streams, third party leagues or competing websites may be emboldened to pilfer from other leagues, and others may elect to stream significant game matches via video, rather than arguably more efficient input streams. In other words, little would be gained, and much would be put at unnecessary risk.

\section{Conclusion}

The granularity offered by an input stream, as fixed in a tangible medium, is sufficient to fully capture the creative expression of gameplay performances and is arguably

128 Ibid.

129 Ibid.

130 Ibid.

131 Dota Team, 'DotaTV Streaming', Dota2.com (January 25, 2018), available at http://blog.dota2.com/2018/01/dotatv-streaming.
25 See generally 'Video Games and the law: Copyright, Trademark, and Intellectual Property', New Media Rights (February 23, 2011), available at right_Trademark_Intellectual_Property.

126 That is, eSports leagues other than those offered by the game publisher or their designee.

127 Maouie Reyes, 'ESL's DMCA Takedowns Draw Ire From Valve and Fans', Mineski.net (January 26, 2018), available at https://www.mineski.net/ news/esl-s-facebook-exclusive-stream-draws-ire-from-fans.

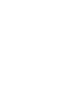


thereby copyright-eligible. Such input stream copyrights should vest in the player, not the creator of the game itself. As strange as it may seem to protect series of keystrokes and mouse clicks, much is to be gained from protecting video game input streams, though the copyright eligibility of an input stream must be no greater than the copyright eligibility of the performance captured.

There are significant benefits to providing a limited copyright over video game-generated input streams. At minimum, third party leagues and players may feel freer to distribute input data, safe with the knowledge that they could stop those misusing input data for unauthorized purposes. And, as discussed above, game companies would not be harmed: they already have a wealth of intellectual property rights at their disposal, all superior to whatever minimal rights correspond to a copyright in an input stream.

Conversely, and as discussed above, if copyright protection is not extended to input streams, at least two negative consequences could arise. First, should individuals (e.g. third-party eSports leagues) continue to use input streams in lieu of video broadcasting for eSports titles, such individuals could quickly find others repurposing their input streams and diverting some of the economic benefits associated with the event. Second, should individuals avoid broadcasting input streams in order to prevent such nefarious behavior, unnecessary time and bandwidth may be used to broadcast gameplay via video instead. Third, to the extent otherwise permitted, third party leagues may be unable to properly monetize the input streams of their work, thereby inhibiting the growth of such leagues.

Various strategies must be explored to proactively assert copyright protection over eSports games. For instance, eSports may benefit from a copyright warning. Major League Baseball famously begins television broadcasts with warnings that the copyrighted telecast 'may not be reproduced or retransmitted in any form, and the accounts and descriptions of this game may not be disseminated without express written consent.'132 While the effectiveness of such warnings is questionable, ${ }^{133}$ the eSports industry must nonetheless explore such strategies as competition becomes increasingly probable.

The beauty of copyright law is that it is flexible, and thus well-positioned to protect the growing eSports industry. eSports is but one issue of many which has arisen as a result of technological developments, and many more are likely to follow. In such circumstances, very little harm is likely to come from providing minimal copyright protection, and yet significant harm may occur should copyright protection not be provided. 